\title{
Symmetry breaking in periodic and solitary gravity-capillary waves on water of finite depth
}

\author{
By JUAN A. ZUFIRIA \\ Applied Mathematics Department, California Institute of Technology, \\ Pasadena, CA 91125, USA
}

(Received 23 September 1986)

A weakly nonlinear model is developed from the Hamiltonian formulation of water waves, to study the bifurcation structure of gravity-capillary waves on water of finite depth. It is found that, besides a very rich structure of symmetric solutions, non-symmetric Wilton's ripples exist. They appear via a spontaneous symmetrybreaking bifurcation from symmetric solutions. The bifurcation tree is similar to that for gravity waves. The solitary wave with surface tension is studied with the same model close to a critical depth. It is found that the solution is not unique, and that further non-symmetric solitary waves are possible. The bifurcation tree has the same structure as for the case of periodic waves. The possibility of checking these results in low-gravity experiments is postulated.

\section{Introduction}

Since early in this century it has been known that the problem of permanent-form gravity-capillary waves travelling on water has a very rich structure of solutions. Wilton (1915) discovered that even for very low-amplitude waves solutions are not unique, i.e. given the shortest period and height of the wave there may be more than one solution. In particular, he studied the case of waves on deep water, and predicted two different wave profiles of $2.44 \mathrm{~cm}$ wavelength. These waves are today called Wilton's ripples. Further, he actually found a set of critical wavelengths where his perturbation solution was not valid.

In the case of gravity waves, it has been shown that for sufficiently low amplitude the solutions are unique. This is because, at low amplitude, resonances are not possible. Two waves are said to be resonant when they travel with the same phase speed. In general, resonances lead to bifurcations into different families of waves and, therefore, to a lack of uniqueness in the solutions. When capillarity is taken into account, the new parameter allows resonances at low amplitude. These can be observed even with linear theory.

Wilton's results were later extended to higher order by Sekerzh-Zenkovich (1956) and Pierson \& Fife (1961) using classical perturbation techniques, and by Nayfeh $(1970 b)$ using the method of multiple scales. Schooley (1961) photographed shortlength wind-generated water waves showing that profiles with two dimples predicted by Wilton (1915) exist. He also showed that, under proper conditions, waves of 3, 4, 5, or more dimples are observed.

Chen \& Saffman $(1979,1980)$ reconsidered the problem of gravity-capillary waves on deep water. They performed perturbation expansions for weakly nonlinear waves and computed finite-amplitude waves using the full water-wave equations. They reinterpreted Wilton's (1915) results as a bifurcation phenomenon and showed that 
higher-order resonances exist and produce bifurcations to new families of solutions with larger wavelengths and more crests per period. Toland \& Jones (1985) and Jones \& Toland (1986) gave rigorous mathematical accounts of the existence theory of the weakly nonlinear waves found by Chen \& Saffman (1980).

Nayfeh $(1970 a)$ considered the problem of periodic gravity-capillary waves on water of finite depth. He obtained third-order expansions for the solution using the method of multiple scales. He found that his expansions broke down for two wavenumbers if the depth of the fluid was larger than some critical value (his condition on the depth of the fluid is equivalent in our notation to the condition $\tau<\frac{1}{3}$, where $\tau$ is defined in (5)). In the limit of deep water Nayfeh's expansion matches Wilton's. The two points where the expansion breaks down correspond to the first two critical wavelengths found by Wilton. Recently Jones (1987), using a functionalanalysis approach based on the Lyapunov-Schmidt reduction procedure, has studied rigorously the existence and multiplicity of small-amplitude solutions of the periodic gravity-capillary wave problem of finite depth. But, as we shall describe below, his work is limited to the study of some particular cases of waves whose shapes are even periodic functions of the horizontal distance (i.e. symmetric waves).

In considering a fluid of finite depth the possibility of solitary-wave solutions is added to the problem. Solitary waves in the presence of surface tension were first considered by Korteweg \& de Vries (1895). They found that for sufficiently large values of the surface tension a depression solitary wave is possible. Recently, Benjamin (1982) and Vanden-Broeck \& Shen (1983) considered the problem again confirming Korteweg \& de Vries results. Amick \& Kirchgässner (1987) have given a rigorous proof of the existence of the solitary wave of depression when $\tau>\frac{1}{3}$. Also Hunter \& Vanden-Broeck (1983) performed numerical computations for the depression solitary wave using the full water-wave equations. They studied the effect of the capillary parameter, and found that, as surface tension was decreased below a critical value $\left(\tau=\frac{1}{3}\right)$, inflexion points appeared in the solitary-wave profile and numerical convergence became very difficult.

Even though there are still many open questions about the problem of Wilton's ripples and solitary waves on finite depth, relatively little work has been done. For gravity-capillary water waves on the surface of the Earth, resonances and all the interesting behaviour appear at depths of approximately $4.8 \mathrm{~mm}$. At this scale the waves are strongly affected by viscosity, so that the irrotational model is not realistic and experimental checks are difficult. Benjamin (1982) tried some experiments with water 3-4 $\mathrm{mm}$ deep, find that depression solitary waves are realizable, but they are damped very rapidly.

With the increasing possibility of performing experiments in outer space, where gravity can be reduced by several orders of magnitude, and the model becomes quite realistic, such experiments are more feasible and make the problem more attractive.

In the present work we shall consider one of the open theoretical questions, namely the existence of non-symmetric gravity-capillary waves. All solutions of permanentform gravity-capillary waves travelling on water known to date describe symmetric waves. A wave is said to be symmetric when, if $f(x)$ represents the shape of the wave, the origin of the horizontal axis can be chosen such that $f(x)=f(-x)$.

Recently, Zufiria $(1987 a, b)$ has shown that non-symmetric gravity waves are possible. They appear via a spontaneous symmetry-breaking bifurcation from symmetric waves. He first found these waves using a weakly nonlinear model for finite-depth water (Zufiria 1987a). Later Zufiria (1987b) extended the results to deep water by numerical computations using the full water-wave equations. It is our belief 
that a great part of the success in extending the results from the model to the full equations comes from using a Hamiltonian formulation of the problem, which keeps the symmetries of the water-wave problem. We found that most of the physical behaviour of the system is related to the Hamiltonian structure and its symmetries.

Motivated by these results, in the present work we develop a weakly nonlinear model for gravity-capillary waves on shallow water. The model is obtained from the Hamiltonian formulation of the water-wave problem introduced by Zakharov (1968). With this model we try to increase the understanding of the Wilton's ripples phenomenon by using dynamical systems theory. In particular we show that non-symmetric waves exist, and they appear via spontaneous symmetry breaking from symmetric waves, as happens for gravity waves.

We start by developing the model, which leads for steady waves to a Hamiltonian system of two degrees of freedom. A local analysis of the solutions around the fixed points of the system is carried out, followed by a numerical global analysis of the model.

In the last part of the work we consider solitary-wave solutions. We give numerical evidence that when the depth of the fluid is close to the resonance value $\left(\tau=\frac{1}{3}\right)$, the solitary wave is not unique and non-symmetric solitary waves are possible.

\section{Weakly nonlinear model}

Consider two-dimensional irrotational water waves in a laterally unbounded domain of constant depth $h$. Taking as variables to describe the state of a wave the shape of the wave $\eta(x, t)$ and the velocity potential evaluated at the surface of the wave $\psi(x, t)=\phi(x, \eta(x, t), t)$, the evolution equations for the system can be written with the following Hamiltonian structure (Zakharov 1968, Broer 1974, Miles 1977):

$$
\frac{\partial \eta}{\partial t}=\frac{\delta \mathscr{H}}{\delta \psi}, \quad \frac{\partial \psi}{\partial t}=-\frac{\delta \mathscr{H}}{\delta \eta},
$$

where $\mathscr{H}$ is the total energy of the waves and $\delta$ represents a functional derivative (see Goldstein 1980, p. 564).

The total energy is defined as

$$
\mathscr{H}=\int H(\eta, \psi) \mathrm{d} x
$$

where the integral extends over one period in the case of periodic waves. In the present study we consider gravity-capillary waves. The energy density $H$ is

$$
H=\frac{1}{2} \int_{-h}^{n}(\nabla \phi)^{2} \mathrm{~d} y+\frac{1}{2} g \eta 2+\frac{T}{\rho}\left\{\left(1+\eta_{x}^{2}\right)^{\frac{1}{2}}-1\right\},
$$

where $g$ is the acceleration due to gravity, $\rho$ the density and $T$ the surface tension.

We consider weakly nonlinear shallow water waves,

$$
\alpha=\frac{a}{h} \ll 1, \quad \beta=\left(\frac{h}{\lambda}\right)^{2} \ll 1,
$$

where $a$ represents a measure of the amplitude and $\lambda$ of the wavelength (order of magnitude of the distance between crests) of the considered wave (see figure 1). The two dimensionless parameters $\alpha$ and $\beta$ measure the amplitude and dispersion of the waves respectively (Whitham 1974).

In order to deal with variables of order unity and determine the relative importance 


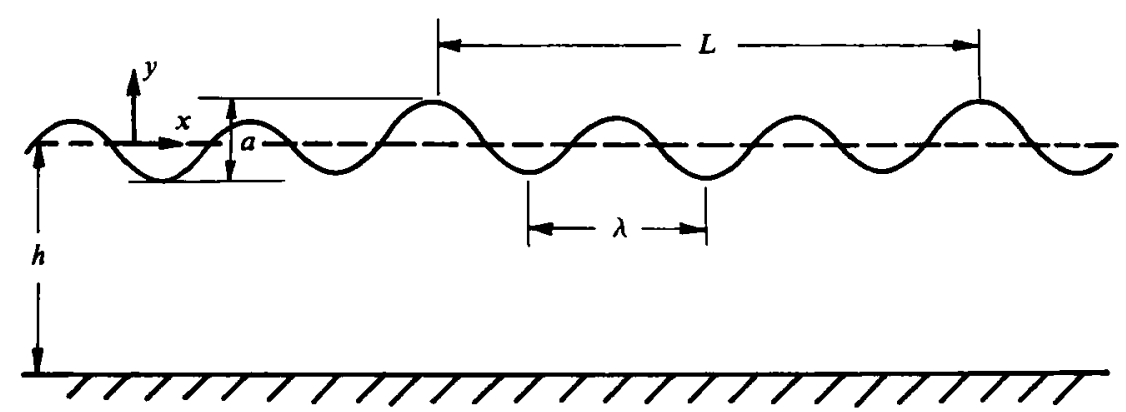

Figure 1. Periodic travelling wave on water of finite depth.

of the different terms in the equations, it is convenient to introduce the following dimensionless variables:

$$
\left.\begin{array}{c}
\bar{x}=\frac{x}{\lambda}, \quad \bar{t}=t\left(\frac{g}{h}\right)^{\frac{1}{2}}, \quad \bar{H}=\frac{\beta \frac{1}{2}}{\alpha^{2}} \frac{H}{g h^{2}}, \quad \bar{y}=\frac{y}{h}, \quad \bar{\phi}=\frac{h \phi}{\lambda a(g h)^{\frac{1}{2}}}, \quad \tau=\frac{T}{\rho g h^{2}}, \\
\bar{\eta}=\frac{\eta}{a}, \quad \bar{\psi}=\frac{h \psi}{\lambda a(g h)^{\frac{1}{2}}} .
\end{array}\right\}
$$

For gravity waves, the nonlinearity is of the same order as the dispersion when the waves are such that $\alpha \approx \beta$. The same is true for gravity-capillary waves except when we look at the minimum of the dispersion. In the region of minimum dispersion, the nonlinearity and dispersion are of the same order when $\alpha \approx \beta^{2}$. Hunter \& Vanden-Broeck's (1983) results showed that this region is where all the interesting resonances and bifurcations occur. Therefore in the following we shall use $\epsilon$ instead of $\alpha$ and $\beta$, where $\epsilon$ is taken as

$$
\alpha=\epsilon^{2}, \quad \beta=\epsilon .
$$

Zufiria (1987a) showed that using the variables introduced in (4) the energy density can be expanded for $\epsilon \ll 1$ as

$$
\begin{aligned}
\epsilon^{\frac{1}{2}} \bar{H}= & \frac{1}{2} \bar{\psi}_{\bar{x}}^{2}\left(1+\epsilon^{2} \bar{\eta}\right)+\frac{1}{2} \bar{\eta}^{2}+\frac{1}{6} \epsilon\left[\bar{\psi}_{\bar{x} \bar{x}}^{2}+2 \bar{\psi}_{\bar{x}} \bar{\psi}_{\bar{x} \bar{x} \bar{x}}\right]+\frac{1}{15} \epsilon^{2}\left[\bar{\psi}_{\bar{x} \bar{x} \bar{x}}^{2}\right. \\
& \left.+\frac{5}{2}\left(\bar{\psi}_{\bar{x} \bar{x}} \bar{\psi}_{x x \bar{x} \bar{x}}+\bar{\psi}_{\bar{x}} \bar{\psi}_{x \bar{x} \bar{x} \bar{x} \bar{x}}\right)\right]+\epsilon_{2}^{1} \tau \bar{\eta}_{\bar{x}}^{2}+O\left(\epsilon^{3}\right) .
\end{aligned}
$$

Using the energy density defined by (7) and neglecting terms of order $\epsilon^{3}$, we obtain the following evolution equations for the water-wave system:

$$
\left.\begin{array}{rl}
\bar{\eta}_{\bar{t}} & =-\bar{\psi}_{\bar{x} \bar{x}}-\frac{1}{3} \epsilon \bar{\psi}_{\bar{x} \bar{x} \bar{x}}-\frac{2}{15} \epsilon^{2} \bar{\psi}_{\bar{x} \bar{x} \bar{x} \bar{x} \bar{x}}-\epsilon^{2}\left(\bar{\eta} \bar{\psi}_{\bar{x}}\right)_{\bar{x}}, \\
\bar{\psi}_{\bar{t}} & =-\bar{\eta}+\epsilon \tau \bar{\eta}_{\bar{x} \bar{x}}-\frac{1}{2} \epsilon^{2} \bar{\psi}_{\bar{x}}^{2} .
\end{array}\right\}
$$

We are looking for permanent-form travelling waves of these equations, i.e. solutions of the form

$$
\left.\begin{array}{c}
\bar{\eta}=\bar{\eta}(\bar{x}, \bar{t})=\bar{\eta}(\bar{x}-c \bar{t})=\bar{\eta}(\bar{x}), \\
\bar{\psi}=\bar{\psi}(\bar{x}, \bar{t})=\bar{\psi}(\bar{x}-c \bar{t})=\bar{\psi}(\bar{x}),
\end{array}\right\}
$$

where $c$ is the phase speed of the wavetrain.

Introducing (9) into (8), we have

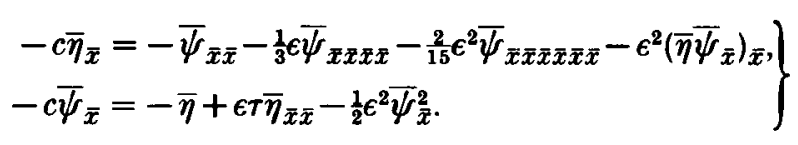


Notice that the linear dispersion relation associated with $(10)$ is

$$
c^{2}=1+\left(\tau-\frac{1}{3}\right) k^{2}+\left(\frac{2}{15}-\frac{1}{3} \tau\right) k^{4}
$$

where $k=2 \pi h / \lambda$ is the wavenumber. This dispersion relation agrees up to (but not including) terms of order $k^{6}$ with the classical dispersion relation for linear water waves, which is

$$
c^{2}=\frac{\tanh k}{k}\left(1+\tau k^{2}\right)
$$

From (11) it follows that system (10) has wavetrains that travel in both directions with phase speeds $c \approx \pm 1$ respectively. To find solutions we shall concentrate our study on waves that travel to the right $(c \approx 1)$.

In the present case of gravity-capillary waves on shallow water resonances appear when $\tau$ is close to $\frac{1}{3}$ (Nayfeh $1970 a, b$; Hunter \& Vanden-Broeck 1983). For $\tau=\frac{1}{3}$ the coefficient of the second term of the dispersion relation (11) vanishes, and the term in $k^{4}$ becomes important; hence the balance between nonlinearity and dispersion leads to waves such that $k^{4} \approx(a / \lambda)$, i.e. $\alpha \approx \beta^{2}$.

Considering waves that travel to the right with $\tau \approx \frac{1}{3}$, a travelling-wave solution can be expanded in the following way:

$$
\left.\begin{array}{rl}
\bar{\psi} & =\bar{\psi}_{0}+\epsilon \bar{\psi}_{1}+\epsilon^{2} \bar{\psi}_{2}+O\left(\epsilon^{3}\right), \\
\bar{\eta} & =\bar{\eta}_{0}+\epsilon \bar{\eta}_{1}+\epsilon^{2} \bar{\eta}_{2}+O\left(\epsilon^{3}\right), \\
c & =1+\epsilon \bar{c}_{1}+\epsilon^{2} \bar{c}_{2}+O\left(\epsilon^{3}\right), \\
\tau & =\frac{1}{3}+\epsilon \bar{\tau}_{1}+\epsilon^{2} \bar{\tau}_{2}+O\left(\epsilon^{3}\right) .
\end{array}\right\}
$$

Substituting these relations into (10), we end up, after some algebra, with

$$
\bar{c}_{1}=0, \quad \bar{\psi}_{0 \bar{x}}=\bar{\eta}_{0}
$$

and the following equation for $\bar{\eta}_{0}$ :

$$
2 \bar{c}_{2} \bar{\eta}_{0 \bar{x}}-3 \bar{\eta}_{0} \bar{\eta}_{0 \bar{x}}+\bar{\tau}_{1} \bar{\eta}_{0 \bar{x} \bar{x} \bar{x}}-\frac{1}{45} \bar{\eta}_{0 \bar{x} \bar{x} \bar{x} \bar{x} \bar{x}}=0 .
$$

This equation can be integrated once with respect to $\bar{x}$ giving

$$
2 \bar{c}_{2} \bar{\eta}_{0}-\frac{3}{2} \bar{\eta}_{0}^{2}+\bar{\tau}_{1} \bar{\eta}_{0 \bar{x} \bar{x}}-\frac{1}{45} \bar{\eta}_{0 \bar{x} \bar{x} \bar{x} \bar{x}}=C \text {. }
$$

The first three terms of the equation represent the Korteweg-de Vries (KdV) approximation.

The origin of $y$ can be chosen such that $C=0$. Thus in the following we assume $C=0$ without any loss of generality, i.e. $\eta=0$ is a solution. Equation (16) can be also directly obtained from the classical water-wave equations by performing the formal expansion for $\epsilon \ll 1$ (Hunter \& Vanden-Broeck (1983).

The dimensionless variables introduced by (5) are useful to determine which terms of the equations are important in the range of waves of interest and to derive the model equation. However, for computational purposes and understanding the nature of the solutions it is more convenient to scale directly in terms of $h$,

$$
\left.\begin{array}{l}
\eta^{\prime}=\frac{\eta}{h}=\epsilon^{2} \bar{\eta}, \quad x^{\prime}=\frac{x}{h}=\epsilon^{\frac{1}{2}} \bar{x}, \\
\tau_{1}=\tau-\frac{1}{3}=\epsilon \bar{\tau}_{1}, \quad c_{2}=c-1=\epsilon^{2} \bar{c}_{2}
\end{array}\right\}
$$

and obtain the equation

$$
2 c_{2} \eta_{0}-\frac{3}{2} \eta_{0}^{2}+\tau_{1} \eta_{0 x x}-\frac{1}{45} \eta_{0 x x x x}=0
$$

where we have dropped the primes. 
In this equation the small parameter $\epsilon$ has disappeard and each term has its own magnitude (i.e. may be large or small depending on the magnitude of the physical wave).

Since $\beta \ll 1, \partial / \partial x \ll 1$ and the last term in (18) can be neglected when $\tau_{1}$ is of order unity, we recover the $\mathrm{KdV}$ approximation. But when $\tau_{1}$ is sufficiently small the fourth term of the equation becomes important.

Equation (18) can be rewritten as the following system of four first-order differential equations:

$$
\left.\begin{array}{l}
\frac{\mathrm{d} u_{1}}{\mathrm{~d} x}=u_{2}, \quad \frac{\mathrm{d} u_{2}}{\mathrm{~d} x}=u_{3}, \quad \frac{\mathrm{d} u_{3}}{\mathrm{~d} x}=u_{4} \\
\frac{\mathrm{d} u_{4}}{\mathrm{~d} x}=\frac{45}{2}\left[\left(2 c_{2}-\frac{3}{2} u_{1}\right) u_{1}+\tau_{1} u_{3}\right],
\end{array}\right\}
$$

where

$$
u_{1}=\eta_{0}, \quad u_{2}=\eta_{0 x}, \quad u_{3}=\eta_{0 x x}, \quad u_{4}=\eta_{0 x x x}
$$

Note that this system is Hamiltonian, with Hamiltonian function

$$
H=\left(c_{2}-\frac{1}{2} q_{1}\right) q_{1}^{2}+p_{1} q_{2}-\frac{1}{2} \tau_{1} q_{2}^{2}+\frac{45}{2} p_{2}^{2} .
$$

and the canonical variables $\left(p_{1}, p_{2}, q_{1}, q_{2}\right)$ related to the variables $\left(u_{1}, u_{2}, u_{3}, u_{4}\right)$ by

$$
\left.\begin{array}{ll}
q_{1}=u_{1}, & q_{2}=u_{2}, \\
p_{1}=\tau_{1} u_{2}-\frac{u_{4}}{45}, & p_{2}=\frac{u_{3}}{45}
\end{array}\right\}
$$

Notice that we started with a continuous Hamiltonian system evolving in time. Looking for travelling waves of that continuous system we finish with a discrete Hamiltonian system that evolves in $x$. The same phenomenon was found by Zufiria (1987a) for gravity waves. Benjamin (1984) has shown that this is a general property for travelling waves of continuous Hamiltonian systems for which the Hamiltonian density can be expressed in terms of finite-order derivatives of the canonical variables, as is the case in our model. Hence, the problem of finding travelling waves of permanent form has been reduced to the study of the properties of a Hamiltonian system of two degrees of freedom with two parameters, $c_{2}$ and $\tau_{1}$.

\section{Local analysis}

The aim of the present study is to understand the structure of the families of periodic orbits of the dynamical system defined by (19). These periodic orbits correspond to travelling waves of permanent form in the physical plane.

We start our analysis by examining the fixed points of the dynamical system and the local structure of the phase flow around them. The two-degrees-of-freedom Hamiltonian system defined by (19) has two fixed points for all values of the parameters $c_{2}$ and $\tau_{1}$. These fixed points are

$$
\left.\begin{array}{ll}
\text { FP1: } & u_{1}=u_{2}=u_{3}=u_{4}=0 \\
\text { FP2: } & u_{1}=\frac{4}{3} c_{2}, \quad u_{2}=u_{3}=u_{4}=0 .
\end{array}\right\}
$$

Both fixed points represent flat surfaces. FP1 is the origin of the phase space and corresponds to the actual surface of the fluid at rest. The other fixed point represents a flat surface also but with a different location of the horizontal axis. The two fixed points represent states of the system that are conjugate (see Benjamin 1984). Notice 


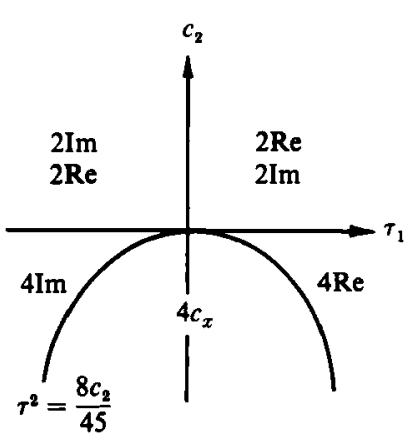

(a)

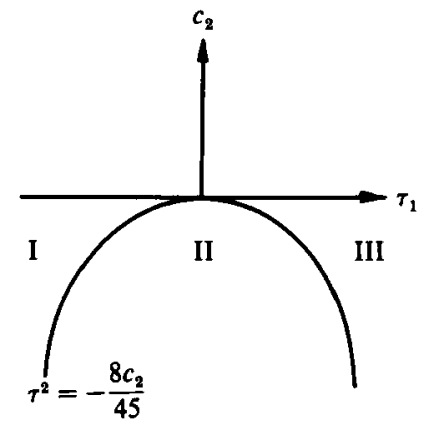

(c)

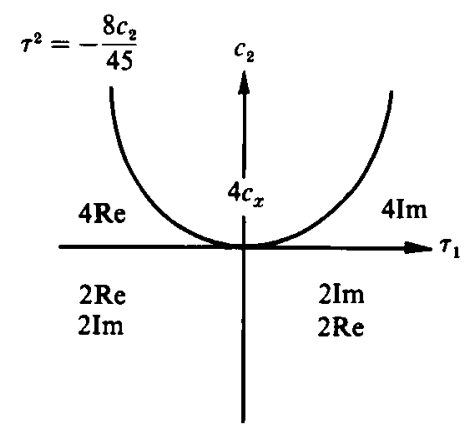

(b)

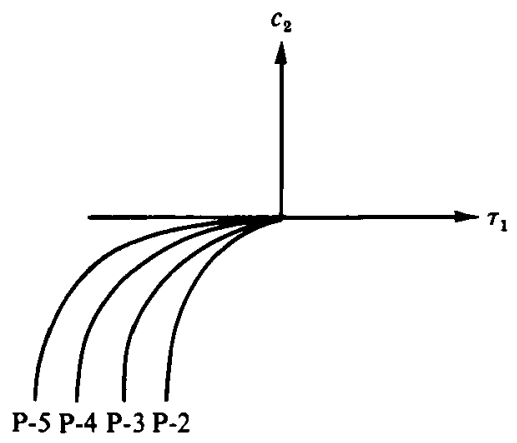

(d)

Figure 2. (a) Eigenvalues for the stability of the fixed point FP1. (b) Eigenvalues for the stability of the fixed point FP2. (c) The three different regions of the $\left(\tau_{1}, c_{2}\right)$-plane where the study is concentrated. $(d)$ Lines where bifurcations ocur at zero amplitude in the $\left(\tau_{1}, c_{2}\right)$-plane. $P_{2}$ denotes period doubling, $P_{3}$ period tripling and so on.

that (19) has two parameters, but the location of the fixed points depends only on one of the parameters, $c_{2}$.

Linearizing the system in the neighbourhood of the fixed points, we find the following eigenvalues for the stability of the fixed points:

$$
\begin{array}{ll}
\text { FP1: } & \lambda^{2}=\frac{45}{2}\left[\tau_{1} \pm\left(\tau_{1}+\frac{8 c_{2}}{45}\right)^{\frac{1}{2}}\right] \\
\text { FP2: } & \lambda^{2}=\frac{45}{2}\left[\tau_{1} \pm\left(\tau_{1}-\frac{8 c_{2}}{45}\right)^{\frac{1}{2}}\right]
\end{array}
$$

For each fixed point we have four eigenvalues, which determine the structure of the fixed point. Depending on the values of the parameters, we can have real, pure imaginary or complex eigenvalues. According to the nature of the eigenvalues the $\left(\tau_{1}, c_{2}\right)$-plane can be divided into four different regions for each fixed point, see figure $2(a, b)$. For example, looking at FP1, we find that for $c_{2}>0$ two of the eigenvalues are real and two pure imaginary. For $c_{2}<0$ there are three different possibilities. If $\tau_{1}<-\left(-8 c_{2} / 45\right)^{\frac{1}{2}}$ there are two pairs of pure imaginary eigenvalues 
$\left(\lambda_{1,2}= \pm i \omega_{1}, \lambda_{3,4}= \pm i \omega_{2}\right)$. For $\tau_{1}^{2}<-8 c_{2} / 45$ the four eigenvalues are complex. And finally, when $\tau_{1}>\left(-8 c_{2} / 45\right)^{\frac{1}{2}}$ all the eigenvalues are real. The situation for FP2 is similar, with $c_{2}$ changed to $-c_{2}$.

According to the previous paragraph and figure $2(a, b)$, there are six different regions in the $\left(\tau_{1}, c_{2}\right)$-plane. But we find that $(18)$ is invariant under the transformation

$$
\begin{aligned}
& c_{2} \rightarrow-c_{2}, \\
& u_{1} \rightarrow u_{1}-\frac{4}{3} c_{2} .
\end{aligned}
$$

This means that the region corresponding to $c_{2}>0$ is equivalent to the region $c_{2}<0$ just interchanging the roles of the two fixed points. Owing to this symmetry we only need to study three of these regions. Therefore, in the following, we reduce the study to the region $c_{2}<0$ (see figure $2 c$ ).

Now consider a local analysis of the three different regions in figure $2(c)$.

Region $I\left(c_{2}<0, \tau_{1}<-\left(-8 c_{2} / 45\right)^{\frac{1}{2}}\right)$

In this region FP1 is a centre. It has two pairs of pure imaginary eigenvalues $\left(\lambda_{1,2}= \pm i \omega_{1}, \lambda_{3,4}= \pm i \omega_{2}\right)$. According to the Liapunov theorem for fixed points of Hamiltonian systems (Abraham \& Marsden 1978), for fixed values of the two parameters $c_{2}$ and $\tau_{1}$ the phase space has two two-dimensional manifolds containing FP1. Each of these manifolds contains a one-parameter family of periodic orbits whose period approaches $2 \pi / \omega_{1}$ or $2 \pi / \omega_{2}$ respectively as the amplitude goes to zero and the orbit approaches the fixed point. This is actually only true when there are no resonance relations between the two frequencies. We say that two frequencies are resonant when there exist two integers $m$ and $n$ such that $m \omega_{1}+n \omega_{2}=0$. In resonant cases linear stability is not enough to determine the local structure of the phase flow around the fixed points.

Note that the Liapunov theorem for Hamiltonian systems is equivalent to the Hopf theorem for generic dynamical systems.

In region I, FP2 has two pure imaginary and two real eigenvalues $\left(\lambda_{1,2}= \pm \mathrm{i} \omega, \lambda_{3,4}= \pm \alpha\right)$. Hence according to the Liapunov theorem there is a single two-dimensional manifold containing FP2 and a one-parameter family of periodic orbits whose period approach $2 \pi / \omega$ as the amplitude goes to zero.

Since there are two families of periodic orbits centred on FP1, this region is very rich from the bifurcation point of view, as we shall see below.

Region $I I\left(c_{2}<0, \tau_{1}^{2}<-8 c_{2} / 45\right)$

Inside the parabola (figure $2 c$ ) FP1 has four complex eigenvalues $\left(\lambda_{1,2}= \pm\left(\alpha_{1}+i \omega_{1}\right), \lambda_{3,4}= \pm\left(\alpha_{2}+i \omega_{2}\right)\right)$. FP1 has two-dimensional stable and unstable manifolds. On each of the manifolds the flow has a spiral structure. In this case, from the local analysis of FP1 we can conclude nothing about possible families of periodic orbits. We can only say that locally there are no families of periodic orbits that end at the fixed point.

There exists the possibility of a homoclinic connection for FP1. If this homoclinic connection exists, it will correspond to a solitary wave with oscillatory tails. We will present below numerical evidence that this kind of connection exists.

For FP2 we find the same situation as the previous case. As there is a pair of pure imaginary eigenvalues, we have a one-parameter family of periodic orbits centred on FP2. 


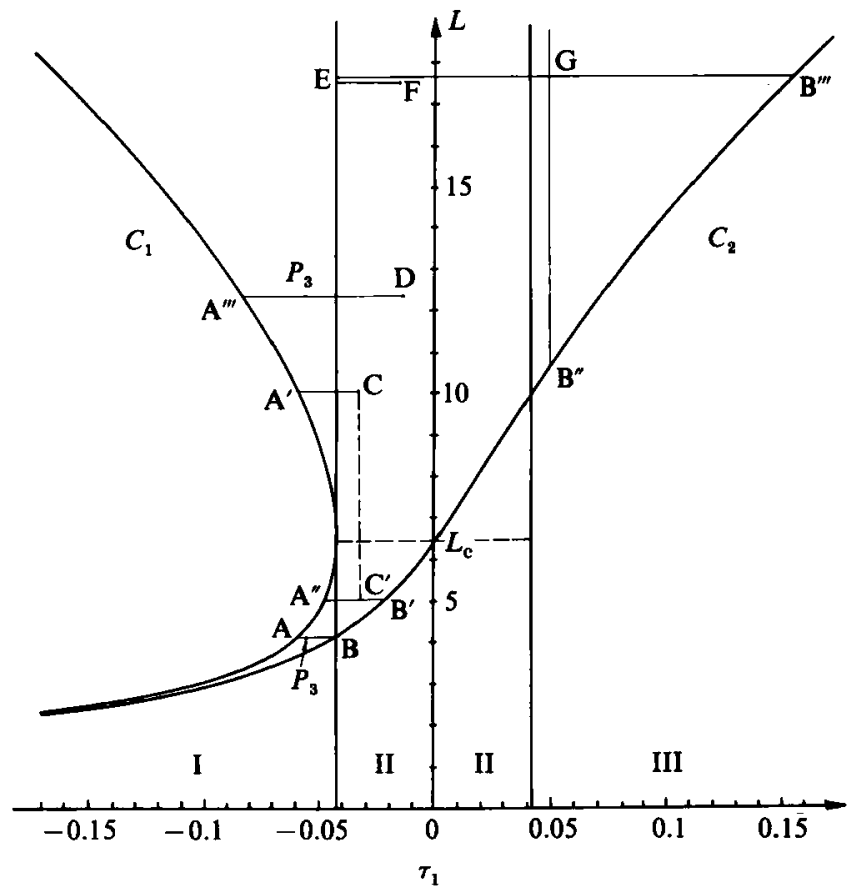

Figure 3. $\left(\tau_{1}, L\right)$-plane. Lines $C_{1}$ and $C_{2}$ show the period of the infinitesimal waves at the fixed points versus $\tau_{1}\left(c_{2}=-0.01\right)$.

Region $I I I\left(c_{2}<0, \tau_{1}>\left(-8 c_{2} / 45\right)^{\frac{1}{2}}\right)$

FP1 has four real eigenvalues $\left(\lambda_{1,2}= \pm \alpha_{1}, \lambda_{2}= \pm \alpha_{2}\right)$. No families of periodic orbits coming from FP1 are possible in this region. On the other hand, FP2 has two real and two pure imaginary eigenvalues. Hence, a one-parameter family of periodic orbits comes from FP2.

Korteweg \& de Vries (1895) showed that solitary waves of depression exist for sufficiently large values of the surface tension. Actually the KdV equation shows that depression solitary waves exist for $\tau>\frac{1}{3}$. The derivation was formalized by VandenBroeck \& Shen (1983) using a systematic perturbation calculation. In the KdV limit, the phase space is two-dimensional and the two fixed points FP1 and FP2 still exist. For $\tau>\frac{1}{3}$ there is a one-parameter family of periodic orbits centred on FP2. FP1 is a saddle (two real eigenvalues).

We expect our results to match with these previous results when the wavelength is increased and $\tau>\frac{1}{3}$. In particular we expect that if the period is increased in the family of periodic orbits that is created at FP2, the family ends up with an orbit that is homoclinic to FP1.

With all these local considerations in mind, we can start the global analysis of the system.

\section{Symmetric waves}

When considering periodic waves of our system for fixed $\tau_{1}$ and a given value of $c_{2}$, the periodic orbits appear as one-parameter families, having the physical period $L$ (the shortest distance over which a solution repeats exactly) as parameter. This degeneracy of the system, owing to its Hamiltonian structure, makes it necessary to 
give three parameters $\left(c_{2}, \tau_{1}, L\right)$ to determine a periodic orbit. Note that in the analysis performed below, when $\tau_{1}$ or $c_{2}$ are changed the phase space is changed. But when $L$ is changed the phase space remains the same and we just move along one of the one-parameter families of periodic orbits that exist in phase space.

We start the global analysis by studying region $I$. As discussed above, in phase space there are two one-parameter families of periodic orbits centred on FP1. These families start at FP1 with periods $2 \pi / \omega_{1}$ and $2 \pi / \omega_{2}$ respectively. If we take $\omega_{1}>\omega_{2}$ then the family corresponding to $\omega_{1}$ has a shorter physical plane period than the other family. We call these families the long-wave family and short-wave family, with respective periods $L_{1}=2 \pi / \omega_{2}$ and $L_{\mathrm{s}}=2 \pi / \omega_{1}$, where $\left(L_{1}>L_{\mathrm{s}}\right) . L_{\mathrm{s}}$ and $L_{1}$ can be regarded as the periods of the infinitesimal waves for fixed $\tau_{1}$ and a given value of $c_{2}$.

The phase speed $c_{2}$ determines the location of the fixed points and gives a measure of the amplitude of the waves. The capillarity parameter $\tau_{1}$ determines for a given fluid and given gravity the depth of the fluid layer.

In the present analysis we fix the phase speed $c_{2}$, and we study the structure of the phase space varying only the capillarity parameter $\tau_{1}$ and the physical period $L$. Using these two parameters, the fixed points FP1 and FP2 are represented in the $\left(\tau_{1}, L\right)$-plane by two lines $C_{1}$ and $C_{2}$ as can be seen in figure 3 . These lines represent, for a given value of $c_{2}$, the initial period of the families of periodic orbits versus $\tau_{1}$, i.e. $L=L\left(\tau_{1} ; c_{2}\right)$. We can see that in region $I$ for a given value of $\tau_{1}, C_{1}$ gives two periods corresponding to the two families that come out of FP1. We can also see that the periods are ordered such that $L_{1}>L_{\mathrm{c}}>L_{\mathrm{s}}$, where

$$
\left(\frac{2 \pi}{L_{\mathrm{c}}}\right)^{2}=\frac{45}{2}\left(\frac{-8 c_{2}}{45}\right)^{\frac{1}{2}} \text {. }
$$

For large period $L$ the two lines $C_{1}$ and $C_{2}$ match asymptotically with the equivalent lines obtained using the $\mathrm{KdV}$ approximation, which is valid for very large wavelengths and away from the resonance region.

To study the possible bifurcations of the families of periodic orbits we need to compute the Floquet multipliers of the orbits. The multipliers are the eigenvalues of the linearized Poincare map of the flow around a periodic orbit. These eigenvalues can be thought as the magnification of a perturbation in one period (Hartman 1964). The necessary and sufficient condition for a bifurcation is that a multiplier crosses the unit circle.

A periodic orbit always has a multiplier +1 corresponding to sliding a little along the orbit. For Hamiltonian systems, the Hamiltonian is conserved so there is another multiplier at +1 . Also in a Hamiltonian system the flow preserves volume in phase space, thus the product of all four multipliers is +1 . It follows, from the reality of the Hamiltonian, that the two other multipliers will be conjugate points $\sigma, \sigma^{*}$ on the unit circle or reciprocal points $\sigma, 1 / \sigma$ on the real axis (Green et al. 1981). When the two multipliers are on the unit circle, we have possible bifurcation to new orbits. For example if $\sigma=\mathrm{e}^{2 \pi \mathrm{i} m / n}$, at that point there is a possible bifurcation to a period- $n$ wave. When $\arg (\sigma) / 2 \pi$ is an irrational number we can still talk about bifurcations but the new branch corresponds to a quasi-periodic permanent-form travelling wave. Notice that from this point of view bifurcations appear within continunous intervals of the parameter values. When approaches that allow only periodic waves, like the ones used by Chen \& Saffman (1980), Toland \& Jones (1985), Jones \& Toland (1986) and Jones (1987), are considered, only the bifurcations that lead to periodic waves can be detected and they appear in a discrete way. The works by Chen \& Saffman (1980) 
and Jones \& Toland (1987) cover the cases where $\sigma=\mathrm{e}^{2 \pi \mathrm{i} n / m}$ for deep-water waves. While Jones (1987) covers the bifurcations of the type $\sigma=\mathrm{e}^{2 \pi \mathrm{i} n /(n+1)}$ for finite-depth water.

Performing a local analysis of the system around FP1, we can compute analytically the Floquet multipliers for the beginning of the families of periodic orbits. We find that for the short-wave family the multipliers at FP1 (zero-amplitude wave) are

$$
\sigma_{1,2}=+1, \quad \sigma_{3,4}=\mathrm{e}^{2 \pi \mathrm{i} \omega_{2} / \omega_{1}} .
$$

Hence the multipliers are on the unit circle, and FP1 is a bifurcation point in this region. This means that we can have bifurcation at zero amplitude. We already knew this because at FP1 two different families of periodic orbits cross. When studying gravity waves Zufiria (1987a) found that the multipliers go onto the unit circle only for high-amplitude waves. Here we have one parameter more, and we can bring the bifurcations to zero amplitude. For example if $\tau_{1}$ and $c_{2}$ are chosen such that $\omega_{2} / \omega_{1}=\frac{1}{2}$ then $\sigma_{3,4}=-1$, and we have a period-doubling bifurcation at zero amplitude. In general $\omega_{2} / \omega_{1}=m / n$ corresponds to a bifurcation from a family of period $L_{\mathrm{s}}$ to a family of period $n L_{\mathrm{s}}$. Notice that this relation is equivalent to a resonance relation. In figure $2(d)$ we show lines where bifurcations occur at zero amplitude.

To understand the global structure of the phase space, we continued numerically the families of periodic orbits by using the program AUTO developed by Doedel \& Kernevez (1986). This program performs continuation along families of periodic orbits for dynamical systems by using a collocation method.

In the present analysis, we kept the value of the phase speed fixed at $c_{2}=-0.01$. We started at the fixed point FP1 by taking $\tau_{1}=-0.06$. These values correspond to region I. Starting from a fixed point there are basically two ways for performing the continuation. One is to continue in the period $L$ keeping the capillarity parameter $\tau_{1}$ constant (vertical lines in figure 3 ). The other possibility is to continue in the capillarity parameter keeping the period constant (horizontal lines in figure 3 ). In the present section we consider the latter case.

We began continuing along the short-wave family coming out from FP1 (point A figures 3 and 4). Following the branch with the period $L$ constant, we found that $\tau_{1}$ increases monotonically and the family ends in the fixed point FP2 (point B figures 3 and 4). Hence the unique family coming out from FP2 is connected to the short-wave family that starts at FP1. Along the whole branch the wave is basically sinusoidal with only one crest per period. The wavelength of this family is always less than $L_{\mathrm{c}}$. This branch is represented in figure 3 by the A-B line.

The long-wave family $\left(L_{1}\right)$ was found to finish in a period-half bifurcation. It starts with a sinusoidal waveform with only one crest per period (point $A^{\prime}$ figures 3 and 4), but as we go along the branch another crest appears. The branch ends when the two crests are equal, which corresponds to a period-half bifurcation (point $\mathrm{C}$ figures 3 and 4). This point corresponds to a period-doubling bifurcation on the short-wave family of period $L_{\mathrm{s}}=\frac{1}{2} L_{1}$ (point $\mathrm{C}^{\prime}$ figures 3 and 4). Notice that this is true only if $\frac{1}{2} L_{1}<L_{\mathrm{c}}$. If this relation does not hold, the structure is much more complicated and will not be considered further here. The three branches of periods $L_{\mathrm{s}}, L_{1}$ and $\frac{1}{2} L_{1}$ are shown in figure 4.

As can be seen in figure 4, along these branches the Floquet multipliers of the orbits go onto the unit circle several times. When the multipliers are on the unit circle, bifurcations to families with larger period are possible. In the following section we study some of these larger-period families. 


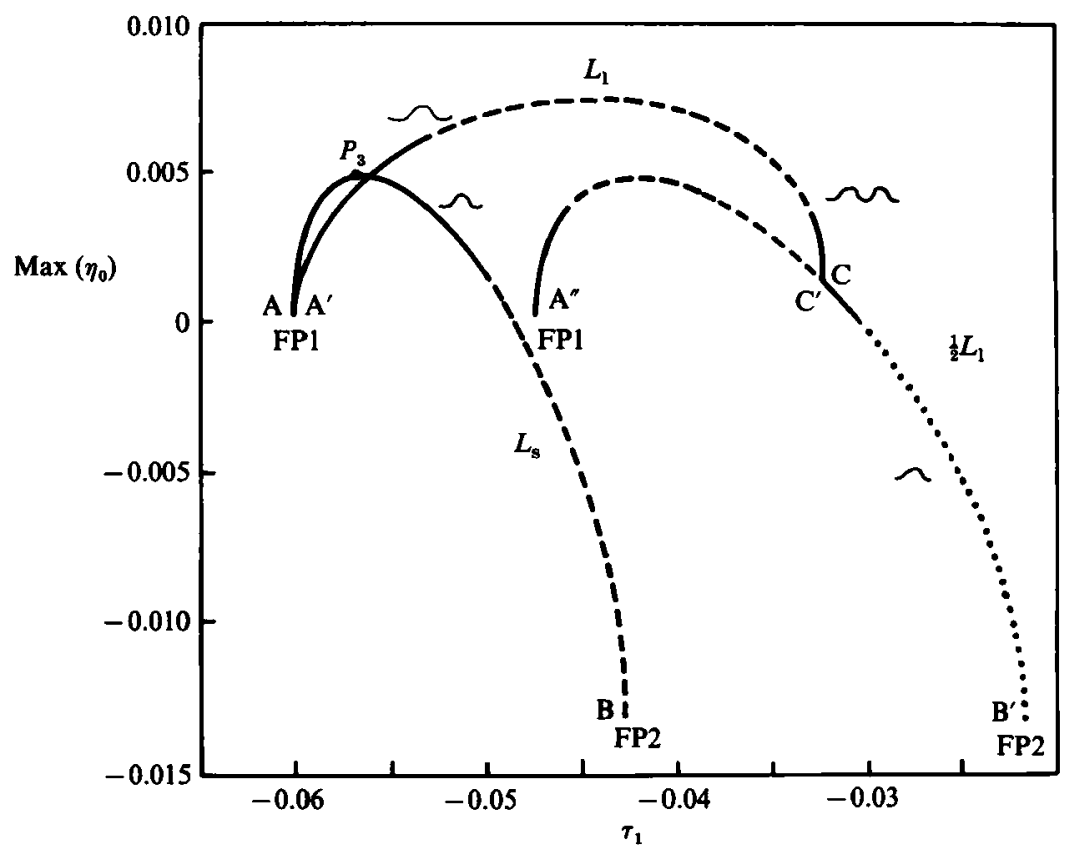

Figure 4. Amplitude of short-wave and long-wave branches of periodic orbits with constant period versus $\tau_{1}$. Solid line denotes $\sigma$ on the unit circle, dashed line $\sigma$ negative and dotted line $\sigma$ positive. Shape of the wave is sketched next to curve.

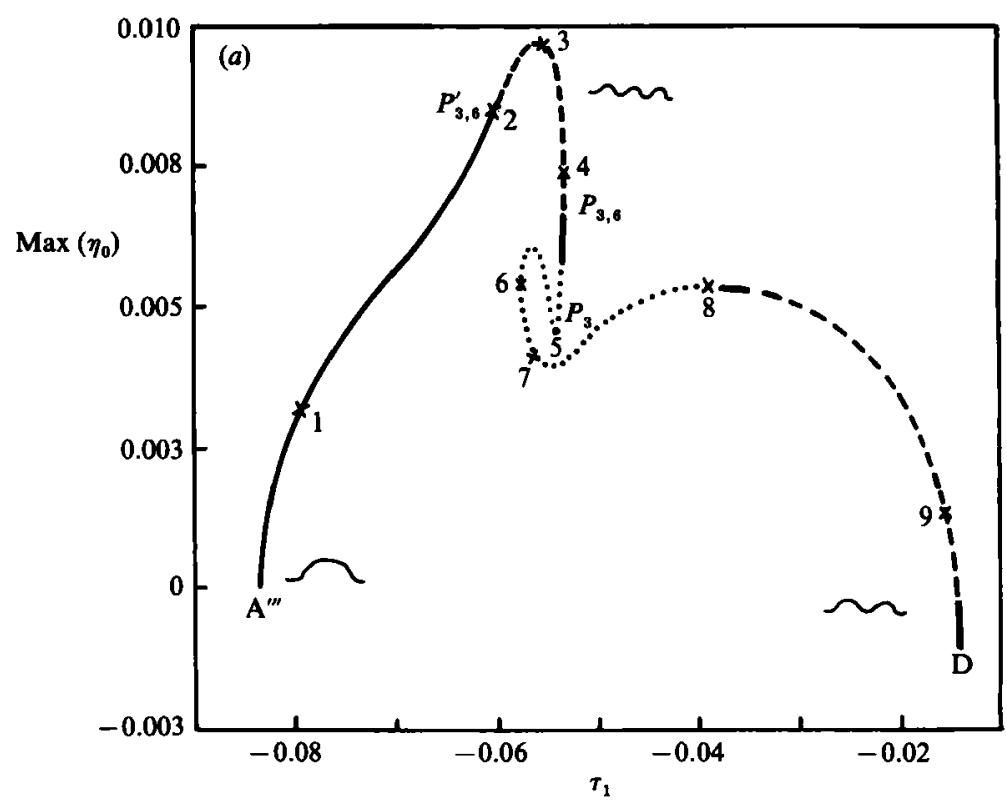

Figure $5(a-b)$. For caption see facing page.

\section{Non-symmetric waves}

All the gravity-capillary waves that we have studied in $\$ 4$, as well as all the ones studied by other authors (Wilton 1915; Nayfeh 1970a, $b$; Chen \& Saffman 1980; Hunter \& Vanden-Broeck 1983) are symmetric.

Recently, Zufiria $(1987 a, b)$ found that for gravity waves on shallow and deep 
(b)
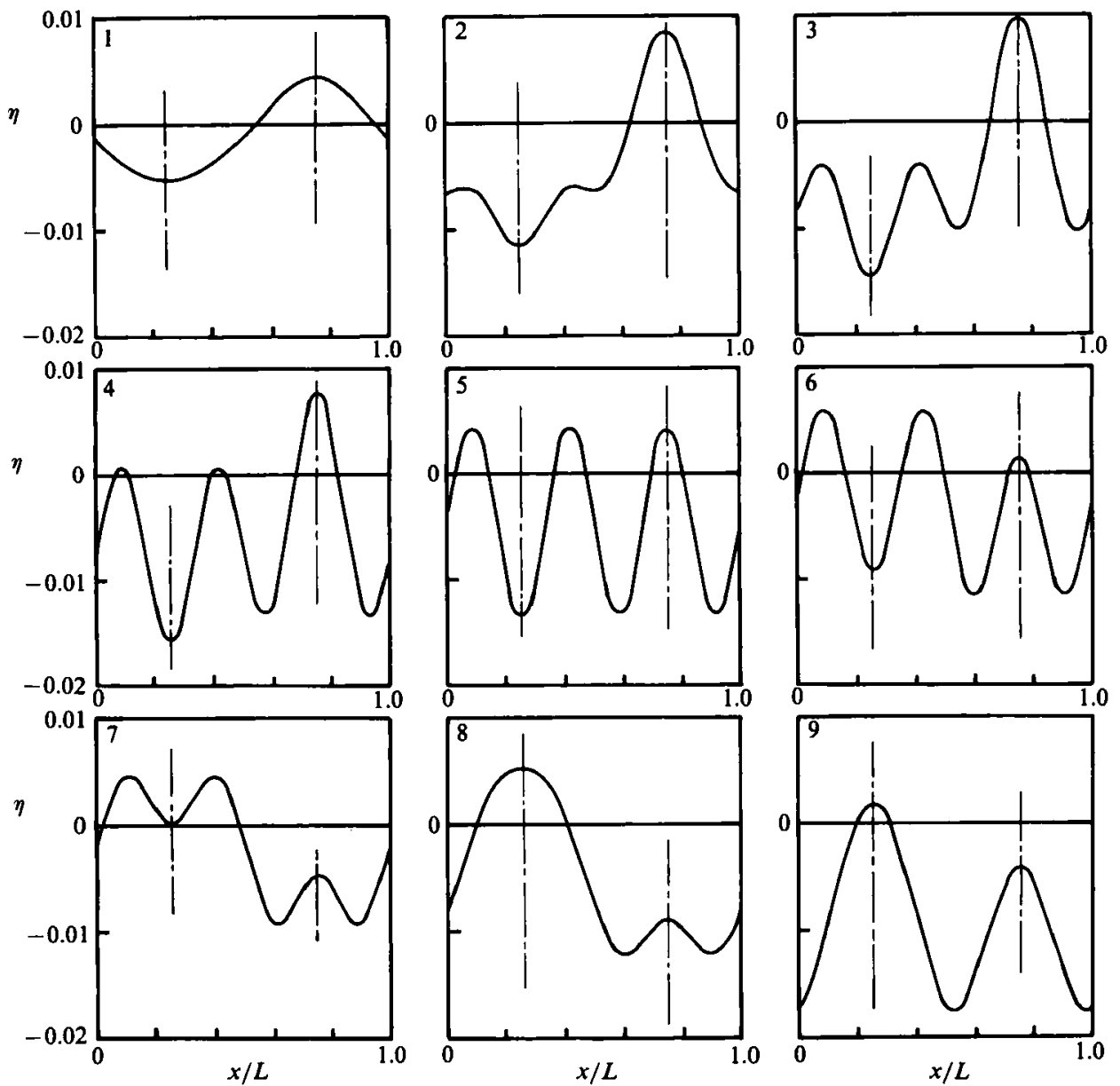

Figure 5. P-3 branch arising from the period tripling $P_{3}$ on branch A-B shown in figure 4. (a) Maximum amplitude of the wave versus $\tau_{1}$ along the branch. (b) Shape of the wave at different points along the branch. The numerals refer to the location of the points shown in $(a)$. Vertical lines show symmetry axes.

water, the symmetry can be broken via a spontaneous symmetry-breaking bifurcation. He found that the sequence of bifurcations that needs to be followed to find the symmetry breaking is the same as that found by Green et al. (1981) for breaking the symmetry of fixed points of area-preserving maps with symmetries. We want to determine if non-symmetric gravity-capillary waves are possible and if the same bifurcation tree is repeated here.

We started again at FP1 and took the short-wave branch. Keeping the period constant we continued in the capillarity parameter $\tau_{1}$ as before. At the beginning of the branch the Floquet multipliers are on the unit circle, and as $\tau_{1}$ is increased they leave the unit circle through -1 (see figure 4). The point where the multipliers are at -1 corresponds to a period-doubling bifurcation to a long-wave family of period $L_{1}=2 L_{\mathrm{s}}$. When the multipliers are at $120^{\circ}$ we have a period tripling (point $P_{3}$ in figures 3, 4 and 5).

We took the new branch of period $3 L_{\mathrm{s}}$, that we call P-3 branch. Keeping the period 


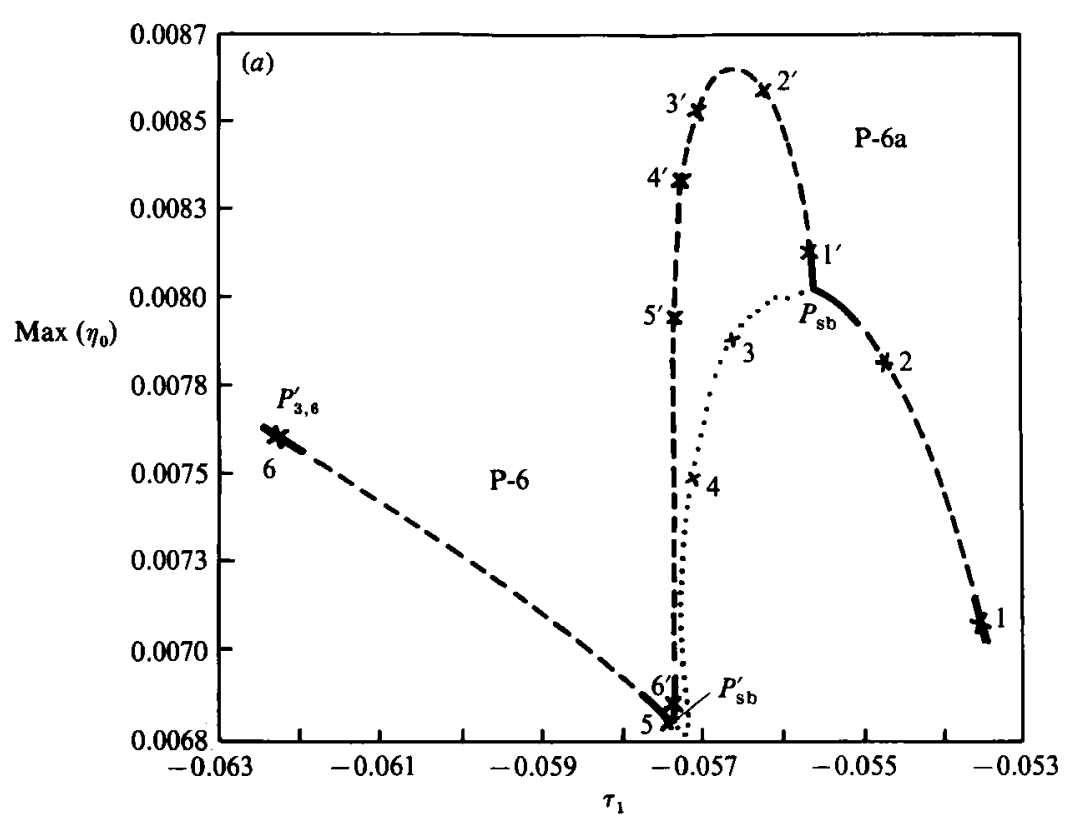

Figure $6(a-c)$. For caption see p. 198.

constant, we continued in $\tau_{1}$ finding that this new branch is just the long-wave branch coming out from FP1 for $L_{1}=3 L_{\mathrm{s}}$. Following the branch in one direction we found that the branch ends in FP1 (point $\mathrm{A}^{\prime \prime}$ figures 3 and 5), and going in the other direction the branch ends in a period-half bifurcation (point D figures 3 and 5 ). The whole branch is plotted in figure $5(a)$. The cusp that appears in the figure because we are plotting the maximum of the amplitude $\eta$. Even though the height of each crest changes smoothly with $\tau_{1}$, the maximum does not change smoothly at the points where our crest becomes higher than another. For example looking at waves 4, 5 and 6 of figure $6(b)$, we can see that on wave 4 the third crest is the highest. Wave 5 corresponds to the point $P_{3}$ where the three crests are equal. And for wave 6 the first two crests are the highest. Hence, the transition through $P_{3}$ is not smooth for maximum of $\eta$. As we can see in the figure the branch is not single valued in $\tau_{1}$. This means that the solution is not unique for a given value of the period and the parameters.

At the beginning of the branch, close to FP1, the wave is sinusoidal and there is only one crest per period. In the region of the non-uniqueness, there are three crests per period. The branch finishes with two equal crests at the half-period bifurcation.

On this P-3 branch, at $P_{3}$ the four multipliers are at +1 as we expected because along this branch $P_{3}$ is a normal bifurcation point. Along the branch there are four regions where the Floquet multipliers lie on the unit circle. Every time that the multipliers leave the unit circle through -1 , we have a period-doubling bifurcation into a family of waves of period $6 L_{\mathrm{s}}$. Following Zufiria $(1987 a)$ we took the new branch of period $6 L_{\mathrm{s}}$ (P-6 branch) that starts at the period-doubling bifurcation closer to $P_{3}$, which corresponds to the point $P_{3,6}$ in figure $5(a)$.

Following figure $6(a)$, starting at $P_{3,6}$ and continuing along the new P-6 branch we found that the branch finishes in a half-period bifurcation, which corresponds to another period doubling (point $P_{3,6}^{\prime}$ in figures 5 and 6 ) of the P-3 branch. The whole branch and the behaviour of the Floquet multipliers along the branch are shown in 
(b)
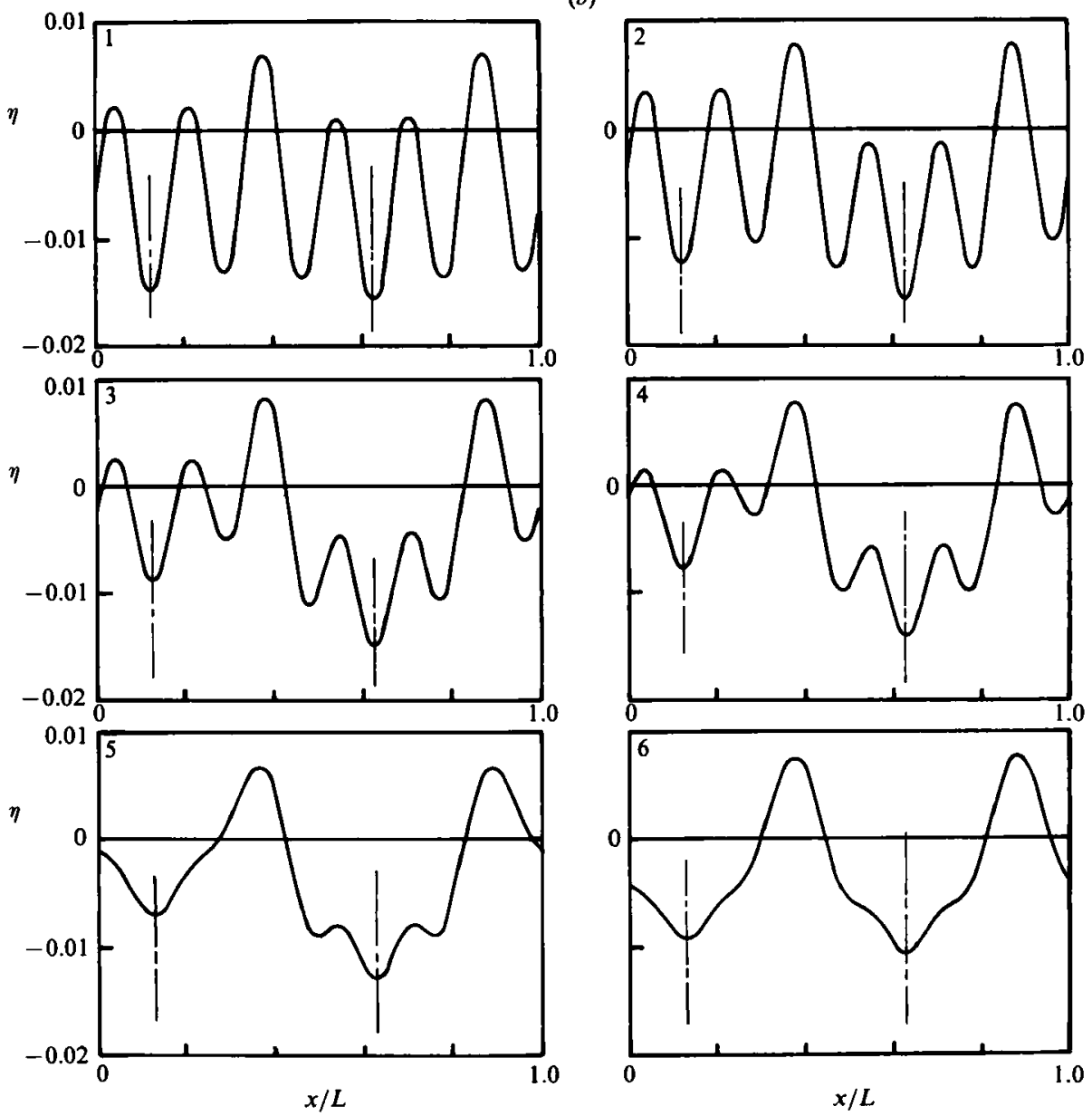

Figure $6(a-c)$. For caption see p. 198.

figure 6. We can see in the figure that the behaviour found by Zufiria $(1987 a)$ for gravity waves appears here again. At $P_{3,6}$ on the P-6 branch the four multipliers are at +1 . As we go along the branch, they move along the unit circle and leave the unit circle through -1 . At this point we have another period-doubling bifurcation to a period $12 L_{\mathrm{s}}$ wave. If we keep going along the P-6 branch the multipliers become negative and $\sigma$ increases in absolute value up to a maximum and starts decreasing again. The multipliers go back onto the unit circle through -1 giving an inverse period doubling. Continuing on the P-6 branch, we find a point where the four Floquet multipliers are at +1 . This kind of bifurcation was studied by Rimmer (1978) (see also Zufiria $1987 a$ ). In the present case we found that the bifurcation is a symmetrybreaking bifurcation.

Starting at $P_{3,6}^{\prime}$ and following the P-6 branch, we find the same structure again. At $P_{3,6}^{\prime}$ the four multipliers are at +1 . As we go along the branch they leave the unit circle through -1 , going back to the unit circle later to give a new symmetry-breaking bifurcation at $P_{\mathrm{sb}}^{\prime}$. Thus, we find two symmetry-breaking bifurcations, points $P_{\mathrm{sb}}$ and $P_{\text {sb. }}^{\prime}$.

Switching to the non-symmetric branch, that we call the P-6a branch, we found 


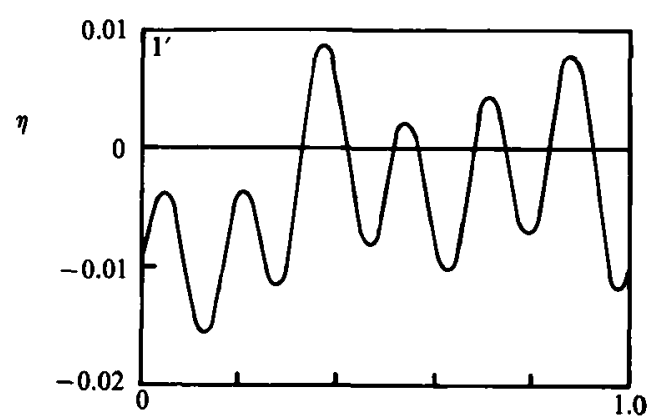

(c)
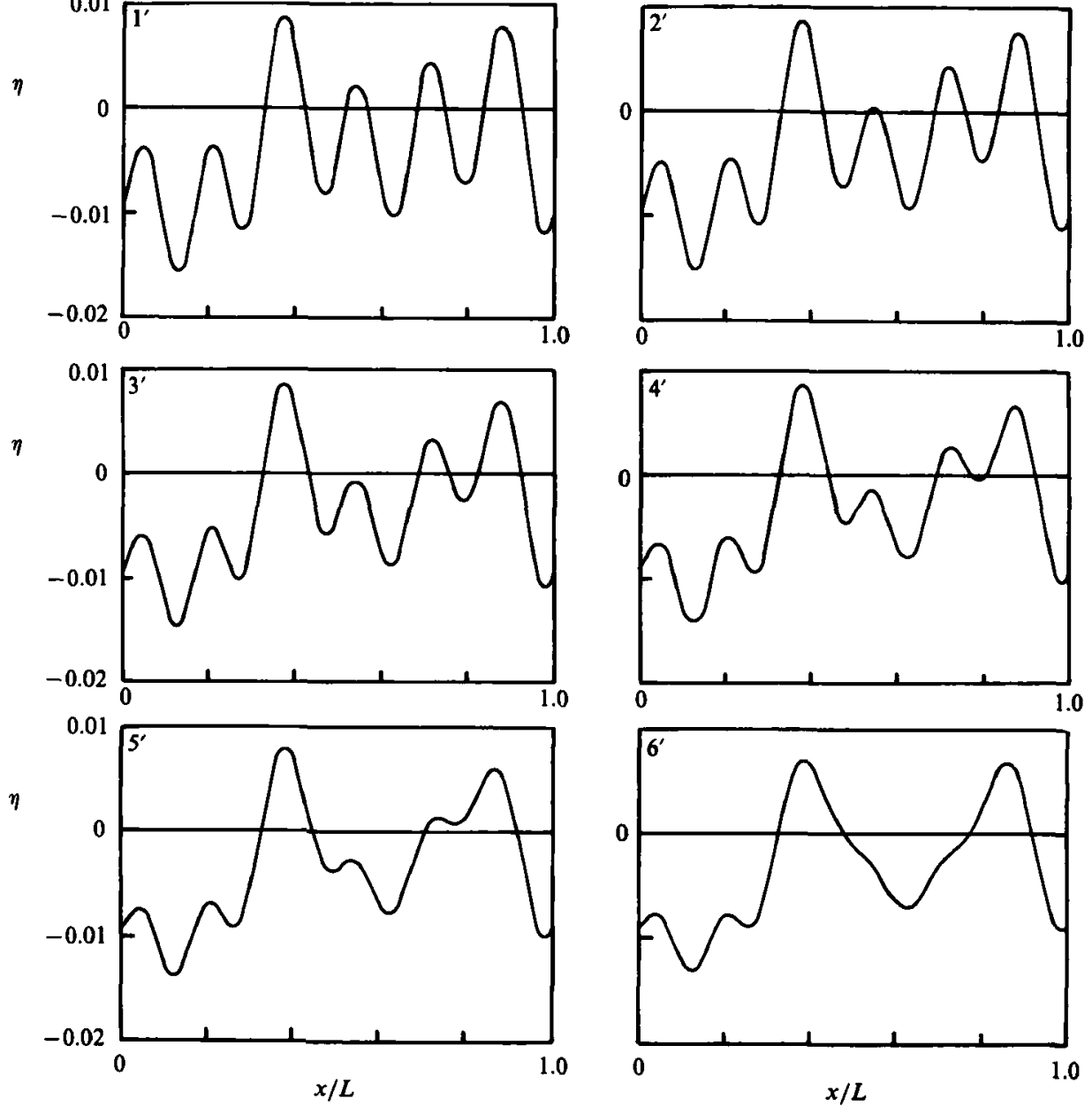

Figure 6. P-6 and P- 6 a branches arising from the period-doubling bifurcations of the P-3 branch shown in figure $5(a)$ and from symmetry-breaking bifurcations of P-6 respectively. (a) Maximum amplitude of the wave versus $\tau_{1}$ along the branches. (b) Plots of the shape of the wave at different points along the P-6 branch. (c) Plots of the shape of the wave along the non-symmetric P-6a branch. The numerals refer to the location of the points shown in $(a)$.

that the branch starts at $P_{\mathrm{sb}}$ and finishes at $P_{\mathrm{sb}}^{\prime}$. So the non-symmetric branch connects the two symmetry-breaking bifurcations.

In figure $6(c)$, we show non-symmetric waves at several points along the branch. We found that, in the present case of gravity-capillary waves, non-symmetric waves of less than six crests are possible. Zufiria $(1987 a, b)$ found that for gravity waves six is the minimum number of crests that is needed to have non-symmetric waves. When capillarity is introduced, crests can appear and disappear along the branches as the capillarity is changed. In the present case, we can even have non-symmetric waves with only two crests.

Keeping $\tau_{1}$ constant we tried to continue in the period with the idea of determining whether solutions with very large period were possible. We found that the period cannot be increased arbitrarily because the branch ends on a symmetry-breaking 


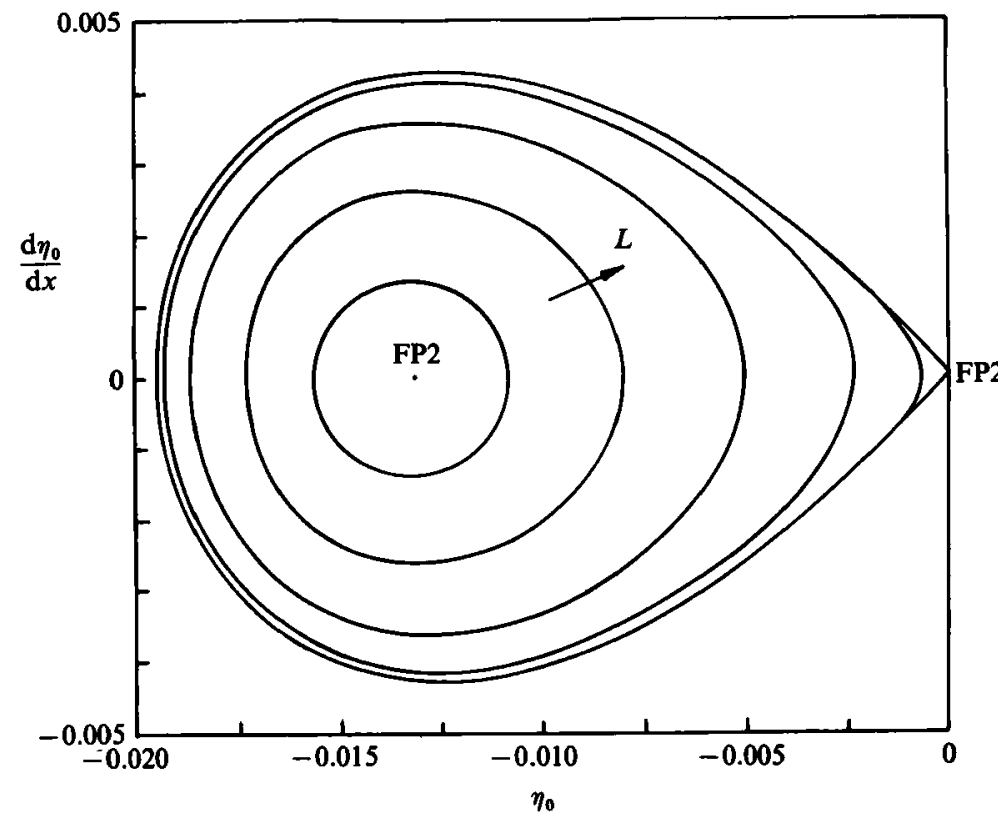

Figure 7. Family of periodic orbits coming from FP2 for $\tau_{1}=0.05$ and $c_{2}=-0.01$ with the period as parameter.

bifurcation similar to $P_{3,6}$. This suggests that non-symmetric solitary waves are not possible in region $I$, and non-symmetric solutions exist only in a bounded domain on the $\left(\tau_{1}, L\right)$-plane.

\section{Solitary-wave solutions}

The problem of solitary waves travelling on finite-depth water with surface tension was first considered by Korteweg \& de Vries (1895). They showed that solitary waves exist for sufficiently large values of the surface tension. The waves that they obtained are actually depression waves. Recently, these results have been rederived by Vanden-Broeck \& Shen (1983) and Benjamin (1982), using a systematic perturbation expansion. These results left open the question of what happens to the solitary wave as the capillarity is decreased. Hunter \& Vanden-Broeck (1983) performed computations for the solitary wave with surface tension using the full water-wave equations. They found that as $\tau$ tends to $\frac{1}{3}$ from above the solitary wave develops a large number of inflexion points, and the computation becomes very difficult. They were not able to compute solutions for $\tau<0.21$ with $c=0.954$ because too many points were required for convergence.

Another apparent result that follows from the studies described above is that for a given value of the capillarity parameter and phase speed or amplitude, there exists a unique solitary wave.

In the present section we use our model to provide evidence that the solitary wave is not unique, and different solutions are possible for the same values of the parameters close to the resonance point, i.e. $\tau \approx \frac{1}{3}$. We also analyse the influence of the capillarity on the solitary wave and the possibility of non-symmetric solutions.

Consider the FP2 point. As already seen in the previous section, for all values of 


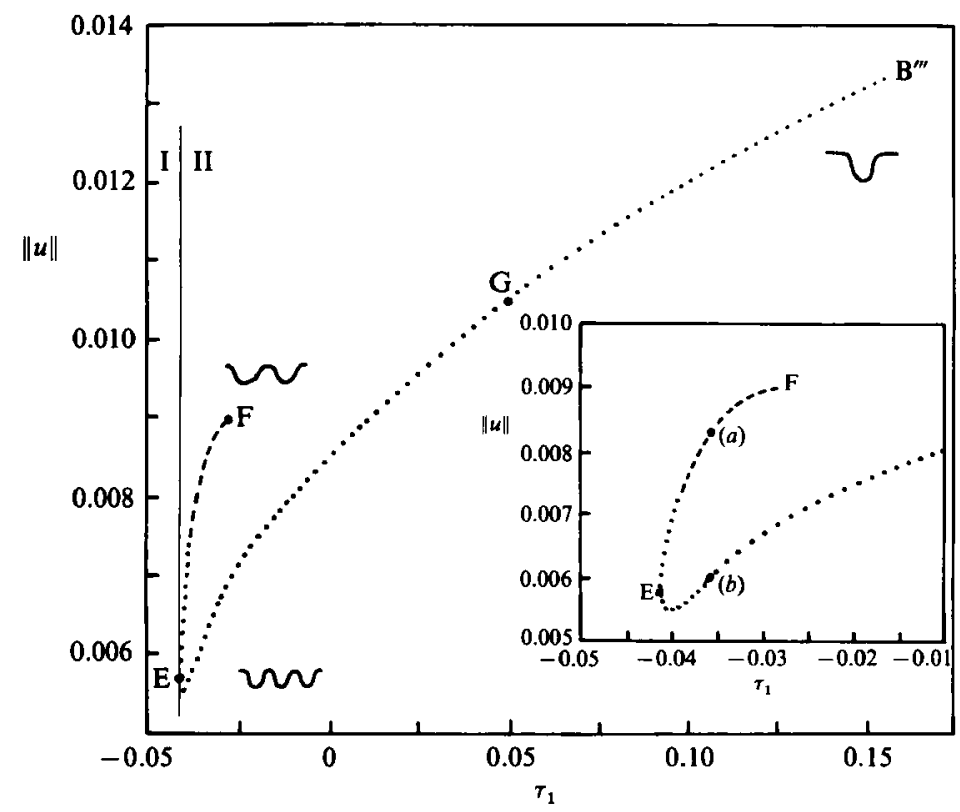

Figure 8. Norm of the solution versus the parameter $\tau_{1}$ for the branch of period $L=17.7$ coming from FP2. Insert shows detail close to the folding point.
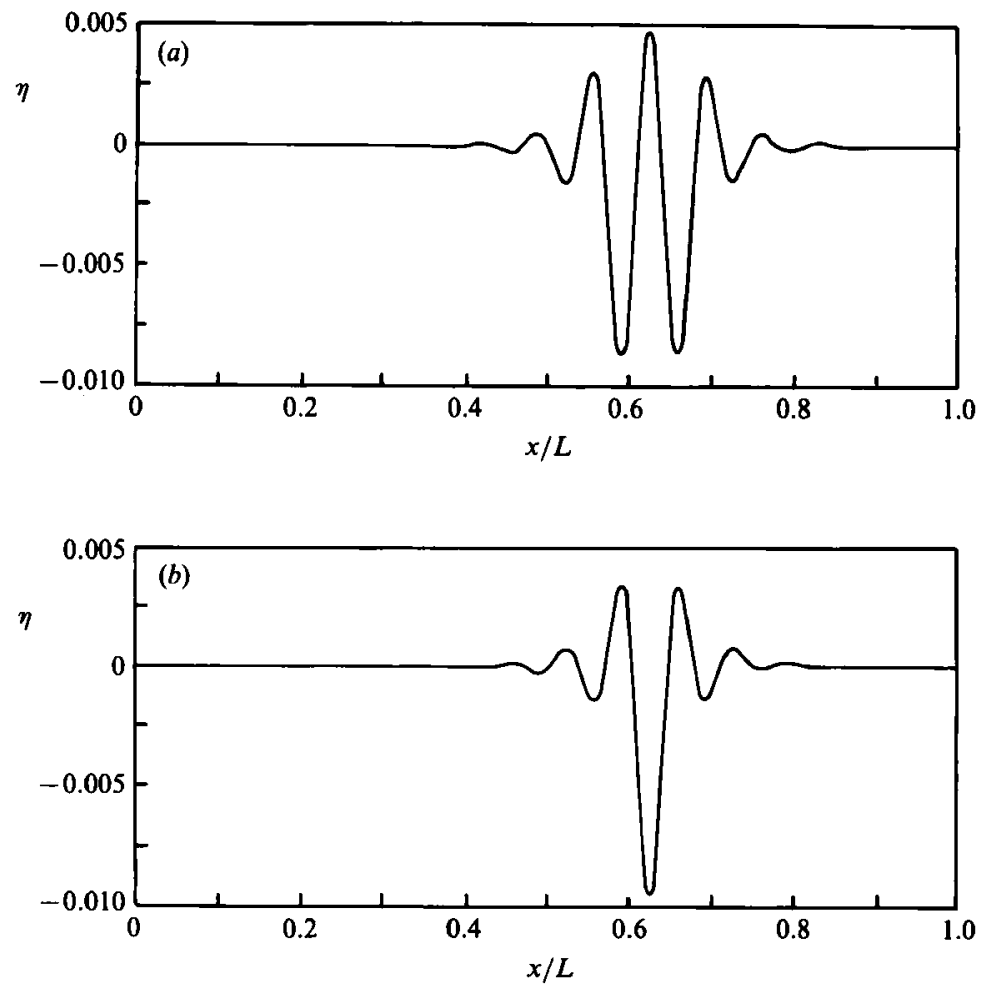

Figure 9. Approximate solitary waves corresponding to points $(a)$ and $(b)$ in figure $8(L=100)$. 
$\tau_{1}$, there is a one-parameter family of periodic orbits coming out from the fixed point. We found that for wavelengths $L$ such that $L<L_{\mathrm{c}}$, as capillarity is changed keeping the period fixed, the family connects the two fixed points. Let us now analyse what happens when larger wavelengths are considered.

We fixed the value of the capillarity parameter at $\tau_{1}=0.05$ and the phase speed at $c_{2}=-0.01$, which corresponds to a point in region III. Starting at FP2 (point $B^{\prime \prime}$ figure 3), we continued along the one-parameter family in the period, i.e. following a vertical path in figure 3 . We found that the family ends up in an orbit that is homoclinic to the origin FP1. This homoclinic orbit corresponds to the depression solitary wave observed by the other authors. In our computations we were able to increase the period of the orbits up to values of order $10^{6}$. We found that the orbit had already converged to the homoclinic one for periods of order $10^{2}$. Hence, we considered this as good evidence that the orbit being computed corresponds to the homoclinic one.

Several orbits of the family are shown in figure 7 . We can see how the homoclinic connection appears as the limiting wave for the family as the period is increased.

In order to find the effect of the capillarity on this family, we took an orbit of the family, that corresponded to a wavelength $L=17.7$ (point $G$ figures 3 and 8 ), and we continued it in the capillarity parameters $\tau_{1}$, keeping the period constant. In figure 8 we plot the norm of the solution versus $\tau_{1}$. The norm that has been used is defined as

$$
\|u\|^{2}=\frac{1}{L} \int_{0}^{L}\left(u_{1}^{2}+u_{2}^{2}+u_{3}^{2}+u_{4}^{2}\right) \mathrm{d} x .
$$

In the direction of increasing capillarity we found, as we already knew, that the family ends in the fixed point FP2 (point $\mathrm{B}^{\prime \prime \prime}$ figures 3 and 8). Decreasing $\tau_{1}$, we first found a fold, a minimum in $\tau_{1}$ (point $E$ figures 3 and 8 ), and after that the branch finishes in a half-period bifurcation (point $\mathrm{F}$ figures 3 and 8 ). Hence for this wavelength, for a given value of $\tau_{1}$ and $c_{1}$, the solution is not unique. This suggests that the solitary wave might not be unique (see figure 8). Therefore we fixed the value of $\tau_{1}$, and we continued in the period for the two different solutions (points $a$ and $b$ in figure 8). We found that both solutions converged to solitary waves, and they were different. Hence depression solitary waves are not unique. In figure 9 we show the two solitary waves that we obtained. Computations were again carried out up to periods of order $10^{6}$, finding again convergence for periods of order $10^{2}$.

Notice that the two homoclinic connections lie in region II, where the origin FP1 has four complex eigenvalues. Therefore we find oscillations at the beginning of the tails of the solitary waves. The number of crests is finite because the real part of the eigenvalues kills the crests and causes the exponentially decaying behaviour. The number of crests increases as the capillarity decreases.

Notice that the minimum of $\tau_{1}$ along the branch is located exactly at the separation point between regions $I$ and $I I$, this being true for whole range of values of $c_{2}$ and $L$. At this minimum, the real part of the eigenvalues of the origin is zero and the solitary wave has an infinite number of crests. These oscillations explain Hunter \& VandenBroeck's (1983) numerical results, and their problems of convergence as capillarity was decreased below $\tau=\frac{1}{3}$.

Going back to the period $L=17.7$ branch and looking at the Floquet multipliers, we found that at FP2 the multipliers are real and positive. As $\tau_{1}$ is decreased we find a point where the four multipliers are at +1 , and after that they become real and positive again. This is the same behaviour as we found on the branch P-3 of the previous section. Examining the solution at this point, we found that the wave has 


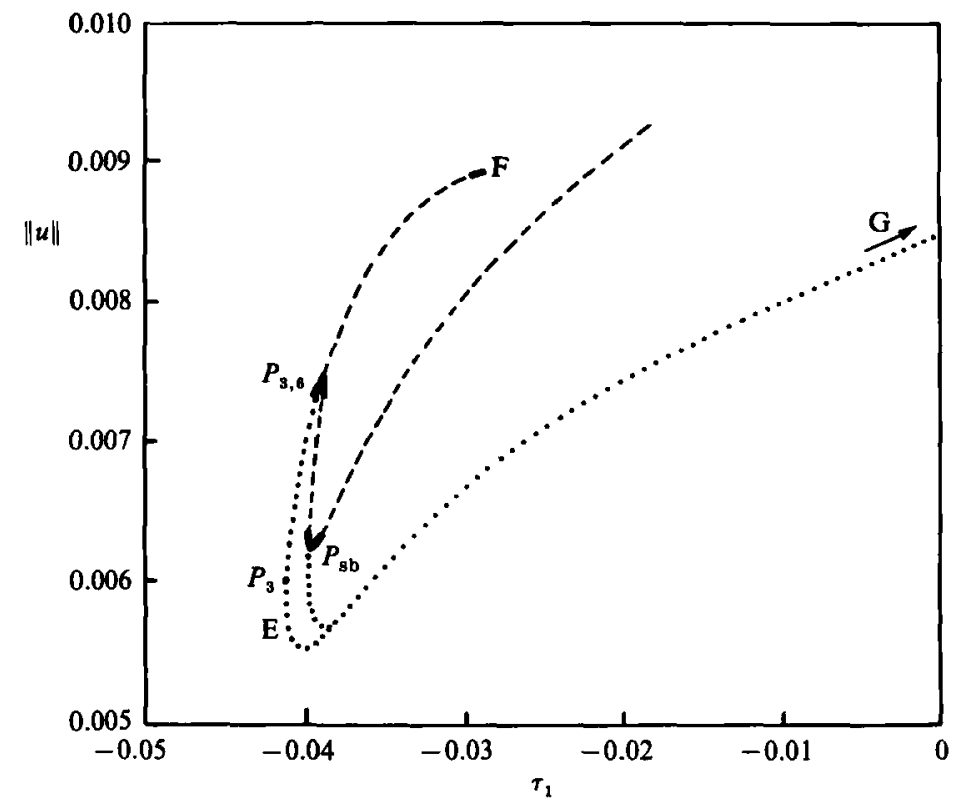

Figure 10. Bifurcation diagram for the branch coming from FP2 $\left(B^{\prime \prime \prime}\right)$ in the neighbourhood of the fold.

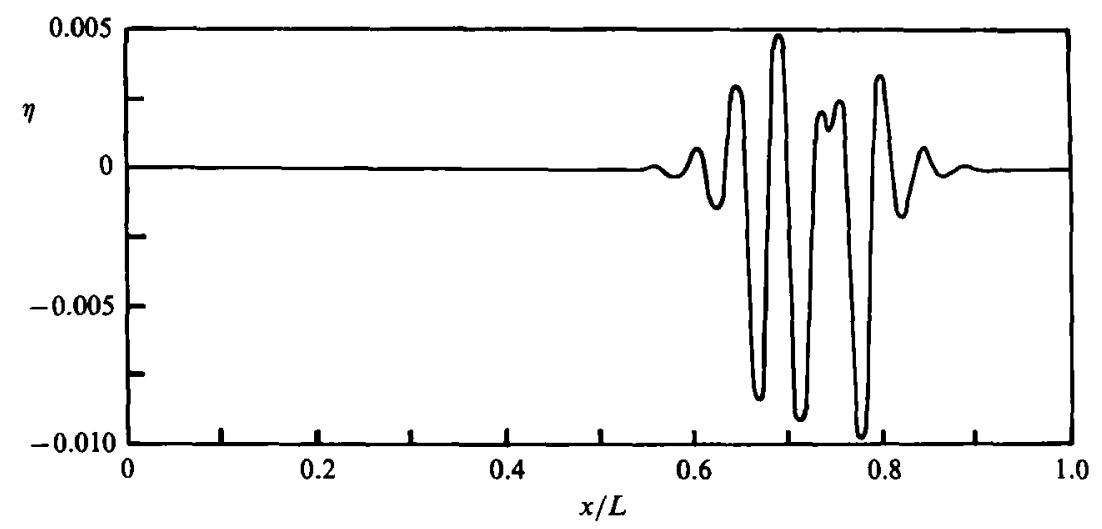

Figure 11. Approximate non-symmetric solitary wave for $L=100, \tau_{1}=-0.039$ and $c_{2}=-0.01$.

three equal crests per period. Thus it corresponds to a period tripling on a branch of period $\frac{1}{3} L=5.9$. The period $\frac{1}{3} L$ branch is a short-wave branch. Recall that in our computations of $\S 5$ we found that on a short-wave branch there is only one period-tripling bifurcation, and this bifurcation gives a wave corresponding to a long-wave branch coming out from FP1. But those computations were performed using a period of $L_{\mathrm{s}}=4.138$. When the period is larger, on the short-wave branch another region appears where the Floquet multipliers are on the unit circle, and therefore there are two period-tripling bifurcations. The new one is the one that we have found in the branch coming out from FP2.

Going along the branch beyond the period-tripling point, we found that the multipliers went onto the unit circle. Again we found the same behaviour as on the 
branch P-3. This suggests the possibility of existence of non-symmetric solitary waves. On this branch where the multipliers leave the unit circle through -1 , we have a period doubling. We took the new branch that corresponds to a wave of period $2 L=35.5$, and computing along it with the period constant, we again found the same behaviour as along the branch P-6 and correspondingly a symmetry-breaking bifurcation. The bifurcation diagram is shown in figure 10. We took the new branch and computed some points on it. For one of these points, we fixed the capillarity and continued in the period, and we found that the orbit converges to a non-symmetric orbit homoclinic to the origin. This orbit corresponds to a non-symmetric solitary wave. In figure 11 we show this approximation to a solitary wave for a period of 100 .

The non-symmetric branch was found to finish on another symmetry-breaking bifurcation, as happened in the case studied in the previous section.

\section{Conclusions}

In the present work, we have developed a weakly nonlinear model to describe low-amplitude and small-dispersion gravity-capillary waves on water of finite depth. The model has been obtained from the Hamiltonian formulation of the water-wave problem. We have found that the analysis of permanent-form travelling waves using this model reduces to the study of the properties of a discrete Hamiltonian system of two degrees of freedom.

Using the dynamical systems theory for Hamiltonian systems, we have studied the bifurcation structure of our model. We have been able to reproduce results on the non-uniqueness of solutions in addition to showing that non-symmetric Wilton's ripples are possible. They appear, as happened in the case of gravity waves (Zufiria $1987 a, b)$, via a spontaneous symmetry-breaking bifurcation. We have found again the same bifurcation tree as the one found by Zufiria (1987a) for finite-depth gravity waves, Zufiria (1987b) for infinite-depth gravity waves and Green et al. (1981) for the fixed point of area-preserving maps in the presence of symmetries. This gives one more example of the universality of this structure in Hamiltonian systems. We have also seen that the symmetric solutions that were known previously are just a subset of the solutions coming from the complex bifurcation structure of Hamiltonian systems.

Numerical evidence is presented showing that solitary waves with sufficiently large surface tension exist. For $\tau>\frac{1}{3}$ the solitary wave is a depression wave. When the surface-tension parameter $\tau$ is decreased below $\frac{1}{3}$, inflexion points appear in the profile of the wave. The tails of the solitary wave start having oscillations, the number of crests being finite. It seems from our results that the solitary wave does not exist in region $I$, i.e. $\tau<\frac{1}{3}-(-8(c-1) / 45)^{\frac{1}{2}}$. For this value of $\tau$, we found a fold in the branch of solutions. This means that the solitary wave is not unique. Actually, we have computed two different solitary waves for the same value of the parameters. Both solitary waves travel with the same speed but have a different crest structure. We have also seen that this is just the beginning of a more complicated structure of solutions. In particular, we have found that a symmetry-breaking bifurcation is possible for the solitary-wave solution. We have computed solitary waves starting from periodic non-symmetric solutions by increasing the period up to very large values $\left(L \approx 10^{6}\right)$. We have found that the solution converges very fast to a non-symmetric solitary wave.

Once again we have found that the bifurcation properties of water waves are related to the Hamiltonian structure of the problem and its symmetries, and that the 
Hamiltonian formulation seems to be a powerful tool to analyse this kind of problem. The stability of all these solutions is an interesting open problem, which we leave as the subject of a future study.

Let us consider the possibility of an experimental check of all these solutions. All these phenomena appear close to the resonance point $\tau=\frac{1}{3}$, which corresponds to a depth

$$
h=\left(\frac{3 T}{\rho g}\right)^{\frac{1}{2}} \text {. }
$$

The typical phase speed $c$ of the waves for this depth is given by

$$
c=(g h)^{\frac{1}{2}}=\left(\frac{3 T}{\rho}\right)^{\frac{1}{4}} g^{\frac{1}{4}}
$$

From Bernoulli's equation it follows that for waves of amplitude $a$ and wavelength $\lambda$, the characteristic velocity $v_{c}$ in the flow is

$$
v_{\mathrm{c}}=\frac{a}{h}(g h)^{\frac{1}{2}}
$$

Given the above definitions, the Reynolds number of the flow can be defined as follows:

$$
R e=\frac{\text { Inertial forces }}{\text { Viscous forces }}=\frac{v_{\mathrm{c}}^{2} / \lambda}{\nu v_{\mathrm{c}} / h^{2}}=\frac{h}{\nu} \frac{a}{\lambda}(g h)^{\frac{1}{2}},
$$

where $\nu$ is the kinematic viscosity. Using (29) the Reynolds number $R e$ can be rewritten as

$$
R e=\frac{a}{\lambda \nu}\left(\frac{3 T}{\rho}\right)^{\frac{3}{4}} g^{-\frac{1}{4}} .
$$

Assuming $\alpha \approx \beta^{2}$ we find that the characteristic frequency in the flow, which is important in order to generate the waves, can be written as

$$
\omega=c k=\frac{2 \pi}{h} \beta^{\frac{1}{2}}(g h)^{\frac{1}{2}}=2 \pi\left(\frac{a}{\lambda}\right)^{\frac{1}{5}}\left(\frac{3 T}{\rho}\right)^{-\frac{1}{4}} g^{\frac{3}{4}} .
$$

Another parameter which gives a good idea of the validity of the irrotational hypothesis and the possibility of performing experiments is the damping coefficient $\gamma$ (Landau \& Lifshitz 1978, p. 100). This coefficient measures the exponential dissipation of the mechanical energy of the wave with time $\left(E \propto \mathrm{e}^{-2 \gamma t}\right)$. For the present case this coefficient is

$$
\gamma=2 \nu k^{2}=8 \pi^{2} \frac{\nu}{h^{2}}\left(\frac{a}{\lambda}\right)^{\frac{2}{5}}=8 \pi^{2} v\left(\frac{\rho g}{3 T}\right)\left(\frac{a}{\lambda}\right)^{\frac{2}{5}} .
$$

In table 1 we show the values of these parameters for four different fluids taking $a / \lambda=10^{-2}$ and two values of the acceleration due to gravity. For an experiment using water on the surface of the Earth we have that $h=4.76 \mathrm{~mm}$, and $c=21.6 \mathrm{~cm} / \mathrm{s}$. The Reynolds number $R e=10$. Evidently, the Reynolds number is too low to neglect viscosity in the model and assume potential flow. As was experimentally checked by Benjamin (1982), the waves are damped too rapidly for an unequivocal investigation of results of an inviscid theory. We can also see that for liquid helium III the situation is a little bit better, but still not satisfactory.

The possibility of performing experiments in a weightless environment in space has completely changed the problem. In a spacecraft the gravity can be reduced by several orders of magnitude, in which case the potential-flow hypothesis is better. For 


\begin{tabular}{llllll}
\hline & Water & Mercury & Helium III & Helium IV \\
& $T / \rho\left(\mathrm{cm}^{3} / \mathrm{s}^{2}\right)$ & 74.0 & 36.8 & 2.5 & 1.9 \\
& $\nu\left(\mathrm{cm}^{2} / \mathrm{s}\right)$ & $10^{-2}$ & $10^{-2}$ & $2.46 \times 10^{-4}$ & $2.31 \times 10^{-4}$ \\
& $h(\mathrm{~cm})$ & 0.475 & 0.335 & 0.088 & 0.077 \\
& $c(\mathrm{~cm} / \mathrm{s})$ & 21.61 & 18.14 & 9.29 & 8.70 \\
& $R e$ & 10 & 6 & 33 & 29 \\
& $\omega(\mathrm{rad} / \mathrm{s})$ & 113.7 & 135.4 & 264.3 & 282.4 \\
& $\gamma\left(\mathrm{s}^{-1}\right)$ & 0.55 & 1.11 & 0.40 & 0.49 \\
& $h(\mathrm{~cm})$ & 14.9 & 10.51 & 2.67 & 2.42 \\
& $c(\mathrm{~cm} / \mathrm{s})$ & 3.86 & 3.24 & 1.66 & 1.55 \\
& $\operatorname{Re}$ & 58 & 34 & 186 & 163 \\
& $\omega(\mathrm{rad} / \mathrm{s})$ & 0.648 & 0.772 & 1.506 & 1.610 \\
& $\gamma\left(\mathrm{s}^{-1}\right)$ & $0.563 \times 10^{-4}$ & $1.134 \times 10^{-4}$ & $0.405 \times 10^{-4}$ & $0.495 \times 10^{-4}$
\end{tabular}

TABLE 1. Values of some parameters for different fluids and different values of the acceleration due to gravity close to the critical point $\tau=\frac{1}{3}$. The values of $T$ and $\nu$ for the helium were taken from Keller (1969).

example if $g=10^{-2} \mathrm{~m} / \mathrm{s}^{2}$, we find that the typical depth on water is $h=14.9 \mathrm{~cm}$ and the phase speed of the waves $c=3.86 \mathrm{~cm} / \mathrm{s}$. For this case $R e=58$. As the gravity is decreased, the Reynolds number increases, and the model is better. The typical length of the problem also increases as the gravity decreases. We find that for liquid helium III $R e=186$ and $h=2.75 \mathrm{~cm}$. It seems from the results shown in table 1 that liquid helium is the best candidate for an experiment among the fluids that we have considered. It seems to be possible to find a compromise solution in the scales, that could make an experiment in space possible.

I am indebted to Professor P. G. Saffman for his encouragement and valuable advice during the course of the research. This work was supported by the Office of Naval Research (N00014-79-C-0412, NR062-639) and by the National Science Foundation (OCE-8415988). I also wish to acknowledge receipt of a Fulbright award.

\section{REFERENCES}

Abraham, R. \& Marsden, J. E. 1978 Fundations of Mechanics, 2nd edn Benjamin/Cummings. AMICK, C. J. \& KIRCHGÄSSNER, K. 1987 On solitary water-waves in the presence of surface tension. (preprint).

Benjamin, T. B. 1982 The solitary wave with surface tension. $Q$. Appl. Maths 40, 231-234.

Benjamin, T. B. 1984 Impulse, force and variational principles. IMA J. Appl. Maths 32, 3-68.

Broer, L. J. F. 1974 On the Hamiltonian theory of surface waves. Appl. Sci. Res. 29, 430-446.

Chen, B. \& Saffman, P. G. 1979 Steady gravity-capillary waves on deep water. I. - Weakly nonlinear waves. Stud. Appl. Maths 60, 183-310.

Chen, B. \& Safrman, P. G. 1980 Steady gravity-capillary waves on deep water. II. - Numerical results for finite amplitude. Stud. Appl. Maths 62, 95-111.

Doedel, E. J. \& K ERNevez, J. P. 1986 Software for continuation problems in ordinary differential equations with applications. Caltech. Applied Maths Rep.

Goldstein, H. 1980 Classical Mechanics, 2nd edn. Addison Wesley.

Green, J. M., MacKay, R. S., Vivaldi, F. \& Feigenbaum, M. J. 1981 Universal behavior in families of area-preserving maps. Physica 3D, 468-486.

Hartman, P. 1964 Ordinary Differential Equations. Wiley-Interscience. 
Hunter, D. H. \& VANDEn-Broeck, J. M. 1983 Solitary and periodic gravity-capillary waves of finite amplitude. J. Fluid Mech. 134, 205-219.

JoNEs, M. C. W. 1987 The bifurcation of Wilton ripples and other capillary-gravity waves in a channel of finite depth. (preprint).

JonEs, M. C. W. \& Toland, J. F. 1986 Symmetry and the bifurcation of capillary-gravity waves. Arch. Rat. Mech. Anal. 96, 29-53.

Kaller, W. E. 1969 Helium-III and Helium-IV. Plenum.

KoRtewe, D. J. \& DE VRIEs, G. 1895 On the change of form of long waves advancing in a rectangular canal and a new type of long stationary waves. Phil. Mag. 39 (5), 422-443.

Landau, L. D. \& Lifshitz, E. M. 1978 Fluid Mechanics. Pergamon.

Mrles, J. W. 1977 On Hamiltonian's principle for surface waves. J. Fluid Mech. 83, 153-158.

Nayfer, A. H. $1970 a$ Finite amplitude surface waves in a liquid layer. J. Fluid Mech. 40, 671-684.

NAYFEH, A. H. $1970 b$ Triple and quintuple-dimpled wave profiles in deep water. Phys. Fluids 13, 545-550.

Pierson, W. J. \& Fife, P. 1961 Some nonlinear properties of long-crested periodic waves with lengths near 2.44 centimetres. J. Geophys. Res. 66, 163-179.

RIMMER, R. 1978 Symmetry and bifurcation of fixed points in area preserving maps. J. Diff Equat. 29, 329-344.

Sekerzh-Zenkovich, Y. I. 1956 On the theory of stationary capillary waves of finite amplitude on the surface of heavy fluid. Dokl. Akad. Nauk. SSSR 109, 913-918.

Schooley, A. H. 1960 Double, triple and higher-order dimples in the profiles of wind-generated water waves in the capillary-gravity transition region. J. Geophys. Res. 65, 4075-4079.

Toland, J. F. \& Jones, M. C. W. 1985 The bifurcation and secondary bifurcation of gravity capillary waves. Proc. $R$. Soc. Lond. A 399, 391-417.

VANDEN-Broeck, J. M. \& Shen, M. C. 1983 A note on the solitary and cnoidal waves with surface tension. $Z$. angew. Math. Phys. 34, 112-117.

WhithaM, G. B. 1974 Linear and Nonlinear Waves. Wiley-Interscience.

Wilton, J. R. 1915 On ripples. Phil. Mag. 29, 688-700.

ZakHAROv, V. E. 1968 Stability of periodic waves of finite amplitude on the surface of deep fluid. J. App. Mech. Tech. Phys. 2, 190-194.

ZUFIRIA, J. A. 1987 a Weakly nonlinear non-symmetric gravity waves on water of finite depth. J. Fluid Mech. 180, 371-385.

ZUFIRIA, J. A. $1987 b$ Non-symmetric gravity waves on water of infinite depth. J. Fluid Mech. 181, 17-39. 